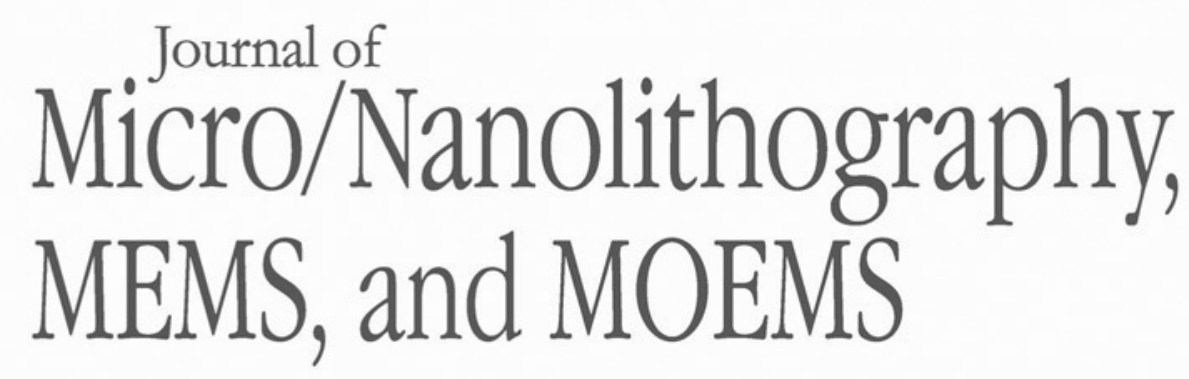

Nanolithography.SPIEDigitalLibrary.org

\title{
2017 List of Reviewers
} SPIE. $\begin{aligned} & \text { "2017 List of Reviewers,” J. Micro/Nanolith. MEMS MOEMS 17(1), } 010102 \text { (2018), } \\ & \text { doi: 10.1117/1.JMM.17.1.010102. }\end{aligned}$ 
The Journal of Micro/Nanolithography, MEMS, and MOEMS would like to sincerely thank the following individuals who served as reviewers in 2017 . The success of our publication hinges on the voluntary contributions of time and energy put forth by these professionals.

Fathi AbdelMalek
Sofia Acosta-Ortiz
Anas Alazzam
Dennis Alexander
James Allen
Tomasz Antosiewicz
Christopher Ausschnitt
Bahram Azizollah Ganji
Sergey Babin
Yasmine Badr
Vadim Banine
George Banke, Jr.
Tan Bo
Robert Brainard
Derk Brouns
Robert Bruce
Timothy Brunner
Martin Burkhardt
James Cameron
Renzo Capelli
Brian Cardineau
Sonia Castellanos
Ayan Chakrabarty
Kin Chan
Glenn Chapman
Chun-Kuang Chen
Tao Chen
Xuanxuan Chen
Yifang Chen
Yulu Chen
Ping Cheng
Seong-O Choi
Yoonsu Choi
Xinyuan Chong
Albert Chu
Anindarupa Chunder
Chris Clifford
Wim Coene
Aaron Cordes
William Cowan
Bo Cui
Gaoliang Dai
Panos Datskos
Guo-Hua Feng
Germain Fenger
Pietro Ferraro
Joter De Binders
Igor Fomenkojov
Claire Deeb
Zhangxian Deng
Dhairya Dixit

Fathi AbdelMalek

Sofia Acosta-Ortiz

James Allen

Tomasz Antosiewicz

Ausschnitt

Sergey Babin

Yasmine Badr

Vadim Banine

Tan Bo

Robert Brainard

Timothy Brunner

Martin Burkhardt

James Cameron

Renzo Capelli

Brian Cardineau

Ayan Chakrabarty

Kin Chan

Tao Chen

Xuanxuan Chen

Ping Chen

Seong-O Cho

Yoonsu Choi

Xinyuan Chong

Albert Chu

Chris Clifford

Wim Coene

Bo Cui

Gaoliang Dai

Panos Datskos

Benyamin Davaji

Peter De Bisschop

Zhangxian Deng

Dhairya Dixit

Yasin Ekinci

Akira Endo

Germain Fenger

Jo Finders

Igor Fomenkov

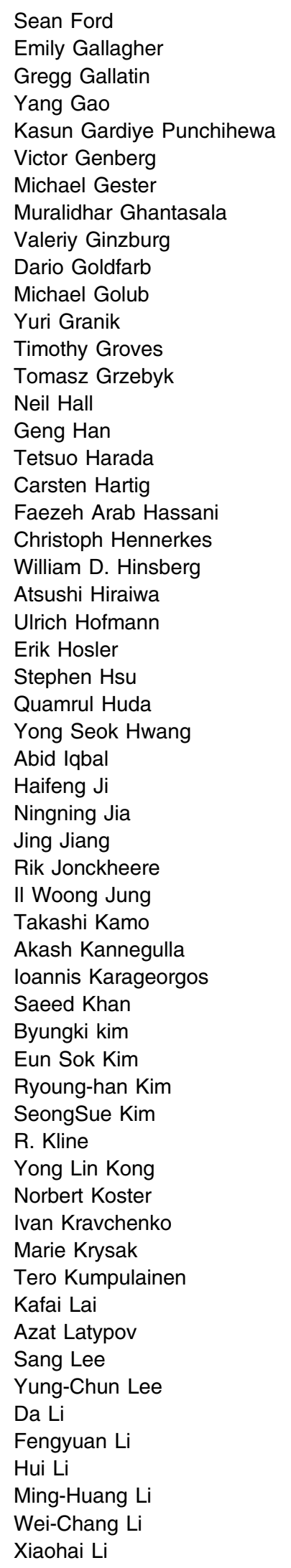

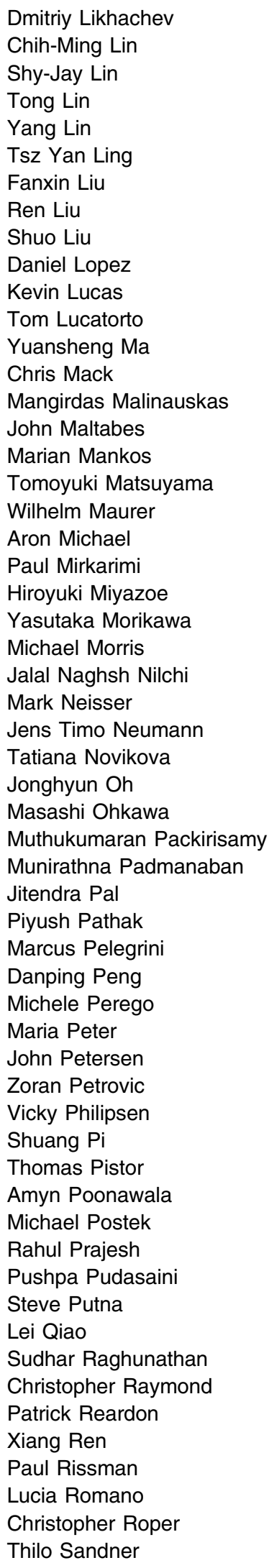


Iqbal Saraf

Cyro Saul

Hella-Christin Scheer

Helmut Schift

Peng Shao

Akshdeep Sharma

Kamran Shavezipur

James Shiely

Renate Sitte

Mark Smith

Robert Socha

John Sturtevant

Zhelin Sun

Kazuaki Suzuki

Kiyoshi Takamasu

Kiwamu Takehisa

Toshihiro Takeshita

Yuksel Temiz
James Thackeray

Marcel Tichem

Kenny Toh

Juan Torres

Peter Trefonas

Jui-che Tsai

Britt Turkot

Takayuki Uchiyama

Alok Vaid

Kaushik Vaidyanathan

Jan van Schoot

Alicia Vera-Marquina

John Villarrubia

Carmen Visconte

Andras Vladar

Christian Wagner

Wentao Wang

Takeo Watanabe
Han Wu

Lingqi Wu

Mingching (Eric) Wu

Wei Wu

Zhengzheng Wu

Jiwen Xiang

Jin Xie

Xin Xie

Yuhao Xu

Bin Yang

Jeehong Yang

Fan $\mathrm{Ye}$

Mao Ye

Frederic Zamkotsian

Ardavan Zandiatashbar

Qiancheng Zhao

Haoshen Zhu

Jie Zou 